\title{
A DFT study of epoxidation of propylene on dimer $\mathrm{MoO}(\mathrm{x})$
}

\author{
Jiahui Peng ${ }^{1}$, Zhehong Wan ${ }^{1}$, Wei Chen ${ }^{2}$, Hui $\mathrm{Hu}^{1}$, Qingming Huang ${ }^{1}$, and Xiaohui Chen ${ }^{1}$ \\ ${ }^{1}$ Fuzhou University \\ ${ }^{2}$ Chinese Academy of Sciences State Key Laboratory of Magnetic Resonance and Atomic \\ and Molecular Physics
}

April 28, 2021

\begin{abstract}
Propylene oxide is an important chemical raw material. In this paper, Density Functional Theory (DFT) was used to calculate the epoxidation of propylene on the dimer MoOx by Gaussian 09 software. Firstly, we established the structure of the dimer $\mathrm{MoOx} / \mathrm{SiO} 2$ and analyzed it, and then calculated the dehydrogenation process of propylene and the formation process of PO. It was found that the activity of $\mathrm{O}$ in Mo-O-Si was higher, which was beneficial to the AHS process of C3H6, but the reaction activity of different $\mathrm{O}$ substances to the formation of PO was very low. In order to solve this problem, we established a dimer MoOx model with defect sites, and found that the defect sites in the dimer could effectively activate O2 (O2- 2), and the activated material $\mathrm{O}$ had high PO selectivity. Compared with process of AHS and the process of PO formation, the energy barrier of PO formation path was very low, which was the main product. At the same time, we also established the MoOx model of Fe doped dimer and the MoOx model of Fe doped defect dimer. It was found that the MoOx clusters were more active due to Fe doping, and the energy barriers of both AHS process and PO formation path were greatly reduced compared with those before doping. The presence of Fe made it easier for the dimer MoOx to form defect sites, which made it easier to activate $\mathrm{O} 2(\mathrm{O}-2)$ and reduced the energy barriers of both AHS and PO formation processes.
\end{abstract}

\section{Hosted file}

A DFT study of epoxidation of propylene on dimer MoOx.pdf available at https://authorea.com/ users/410611/articles/519922-a-dft-study-of-epoxidation-of-propylene-on-dimer-moo-x 